\title{
Apparent Strain Localization and Shear Wave Dispersion in Elastic Fault Gouge with Microrotations
}

\author{
E. Pasternak ${ }^{1,2}$, H.-B. Mühlhaus ${ }^{3}$ and A.V. Dyskin ${ }^{1}$ \\ ${ }^{1}$ School of Civil and Resource Engineering, The University of Western Australia \\ 35 Stirling Highway, Crawley, WA, 6009, Australia \\ Elena@civil. uwa.edu. au \\ ${ }^{2}$ Institut für Werkstoffkunde und Werkstofftechnik, Technische Universität Clausthal \\ Agricolastr. 6, D-38678, Clausthal-Zellerfeld, Germany \\ ${ }^{3}$ CSIRO Division of Exploration and Mining, Australian Resource Research Centre \\ PoBox 1130, Bentley, WA 6102, Australia \\ and Department of Earth Sciences, The University of Queensland \\ St Lucia, QLD 4072, Australia \\ hans.muhlhaus@csiro.au
}

\begin{abstract}
Shear deformation of fault gouge or other particulate materials often results in observed strain localization, or more precisely, the localization of measured deformation gradients. In conventional elastic materials the strain localization cannot take place therefore this phenomenon is attributed to special types of non-elastic constitutive behaviour. For particulate materials however the Cosserat continuum which takes care of microrotations independent of displacements is a more appropriate model. In elastic Cosserat continuum the localization in displacement gradients is possible under some combinations of the generalized Cosserat elastic moduli. The same combinations of parameters also correspond to a considerable dispersion in shear wave propagation which can be used for independent experimental verification of the proposed mechanism of apparent strain localization in fault gouge.
\end{abstract}

\section{Introduction}

Strain localization under shear loading is often observed in granulate materials such as the material of fault gouge. If however one models a fault gouge as a homogeneous elastic layer of thickness $2 h$, occupying the strip $-h<x_{2}<h$ in a Cartesian coordinate frame $\left(x_{1} x_{2} x_{3}\right)$ with specified displacement at the boundaries $u_{1}\left(x_{1}, \pm h, x_{3}\right)= \pm u_{1}{ }^{0}$, $u_{2}\left(x_{1}, \pm h, x_{3}\right)=u_{3}\left(x_{1}, \pm h, x_{3}\right)=0$, then the shear strain and stress will be uniform i.e. $\varepsilon_{12}=u_{1}{ }^{0} / 2 h, \sigma_{12}=\mu u_{1}^{0} / h$, where $\mu$ is the shear modulus of the gouge. Thus the strain localization is perceived as a manifestation of material instability which can only be achieved under either large deformations or certain inelastic constitutive laws, such as non-associated plasticity (eg, [1]) or post-peak softening.

An important feature of granulate materials overlooked by the conventional continuum mechanics is the presence of microrotations arising from the ability of particles to rotate independently, i.e. not in a chord with the rotations associated with the displacement field. This feature can be modelled by Cosserat continuum in which strain and displacement gradient become different. Observations of localization usu- 
ally refer to the measurements of displacement gradients. In the present paper we show that in a Cosserat continuum localization of the displacement gradient can be observed even for elastic gouge. We show that the combinations of parameters producing the localization also lead to dispersion in shear wave propagation.

\section{Three-Dimensional Continuum Elastic Model of Granulate Materials}

Cosserat continuum models of a particulate material can be obtained by homogenisation of discrete equations of motion of the particles. Mühlhaus and Oka [2] suggested a continuum model for assemblies of identical spheres without resistance to relative rotations. The equations of motion were homogenised by expanding the difference expressions of the discrete model into Taylor series and retaining terms up to the second order. Since rotational degrees of freedom were introduced and higher order terms kept in the corresponding Taylor expansions, the resulted continuum description was a combination of a Cosserat theory and a strain gradient theory. An analysis of simple $1 \mathrm{D}$ particle arrangements $([3,4])$ showed that the resistance to relative rotations at particle contacts is important: its neglect leads to the loss of positive definiteness of the energy [2]. In the following we consider the resistance of the spheres to relative rotations at the contact points (see also [5]). The non-symmetry of the stress can then be balanced by moment stresses caused by relative rotations at the contacts alone.

We consider a three-dimensional assembly of identical spherical grains. The diameter $D$ of the grain is assumed as much smaller than the problem dimension $L$, so that $D<<L$. The spheres are in permanent contact; and the orientation of the contact points is assumed as random. In our idealised model, every point of the equivalent continuum corresponds to the centroid of the reference sphere in the discrete material.

Interaction between each pair of neighbouring particles is represented by the total contact force $\vec{F}$ and contact moment $\vec{M}$. The contact moment reflects the fact that the real particles are neither absolutely rigid nor perfectly spherical and hence contact over a certain area. Then the relative rotation of one particle with respect to the other results in a non-symmetric distribution of contact forces, which in the first approximation can be described by the contact moment. It is supposed that the contact force and the moment are linearly dependent upon the relative displacement $\Delta \vec{u}$ and rotation $\Delta \vec{\varphi}$ between the neighbouring particles respectively:

$$
\begin{gathered}
\vec{F}=K \Delta \vec{u}, \quad \vec{M}=L \Delta \vec{\varphi} \\
K=\left[K_{i j}\right], K_{i j}=\left(k_{n}-k_{s}\right) n_{i} n_{j}+k_{s} \delta_{i j}, L=\left[L_{i j}\right], \\
L_{i j}=\left(k_{\varphi_{n}}-k_{\varphi_{s}}\right) n_{i} n_{j}+k_{\varphi_{s}} \delta_{i j} .
\end{gathered}
$$


Here $K$ and $L$ are the matrixes of the translational and rotational spring stiffnesses, $k_{n}$, $k_{s}$ and $k_{\varphi n}, k_{\varphi s}$ are the normal and shear (tangential) contact stiffnesses of the translational and rotational springs and the indices in (2) refer to a spatially fixed Cartesian coordinate system.

Assuming that the particle arrangements are statistically homogeneous and applying the method of homogenisation by differential expansions [4] one obtains the following state and constitutive equations

$$
\begin{gathered}
\sigma_{j i, j}=\rho \ddot{u}_{i}, \quad \mu_{j i, j}+\varepsilon_{i j k} \sigma_{j k}=\rho \frac{D^{2}}{10} \ddot{\varphi}_{i} . \\
\sigma_{j i}=C_{i j l m} \gamma_{l m}+C_{l j} \gamma_{l i}, \quad \mu_{j i}=D_{i j l m} \kappa_{l m}+D_{l j} \kappa_{l i},
\end{gathered}
$$

Here $\sigma_{i j}$ and $\mu_{i j}$ are non-symmetric stresses and moment stresses respectively, $\gamma_{i j}, \kappa_{i j}$ are the classical Cosserat continuum deformation measures (eg, [6])

$$
\gamma_{j i}=u_{i, j}-\varepsilon_{k j i} \varphi_{k}, \quad \kappa_{j i}=\varphi_{i, j}
$$

where $\varphi_{i}$ is the Cosserat rotation, $\gamma_{j i}$ and $\kappa_{j i}$ are strains and curvature twists.

The parameters of the constitutive relationships (4), the elastic moduli $C_{i j l m}, C_{l j}$, $D_{i j l m}, D_{l j}$ have the form

$$
\begin{gathered}
C_{i j l m}=\frac{6 v_{s}}{\pi D}\left(k_{n}-k_{s}\right) A_{i j l m}, C_{l j}=\frac{6 v_{s}}{\pi D} k_{s} A_{l j}, \\
D_{i j l m}=\frac{6 v_{s}}{\pi D}\left(k_{\varphi_{n}}-k_{\varphi_{s}}\right) A_{i j l m}, D_{l j}=\frac{6 v_{s}}{\pi D} k_{\varphi_{s}} A_{l j}, \\
A_{l j}=\int_{\alpha / 2} A n_{l} n_{j} d n=\frac{k}{6} \delta_{l j}, \\
A_{i j l m}=\int_{\alpha / 2} A n_{i} n_{j} n_{l} n_{m} d n=\frac{k}{30}\left\{\delta_{i j} \delta_{l m}+\delta_{i l} \delta_{j m}+\delta_{i m} \delta_{j l}\right\} .
\end{gathered}
$$

Here $A(\mathbf{r}, \mathbf{n})=k / 4 \pi$ for isotropic distribution of particle contacts, $\alpha$ is the spherical angle, $d n=\sin \theta d \theta d \phi$ in a spherical coordinate frame with the origin at the sphere cen$\operatorname{tre}(r, \phi, \theta)$.

\section{Simple Shear of Elastic Gouge with Microrotations}

We consider a model of fault gouge as an infinite Cosserat elastic layer occupying the area $\left|x_{1}\right|<\propto,\left|x_{2}\right|<h$ under plain strain conditions (Fig. 1) subjected to the following boundary conditions: 


$$
u_{1}\left(x_{1}, \pm h, x_{3}\right)= \pm u_{1}^{0}, u_{2}\left(x_{1}, \pm h, x_{3}\right)=0, \varphi_{3}\left(x_{1}, \pm h, x_{3}\right)=0 .
$$

The Cosserat continuum Lamé equations for the plain strain with no body forces and moments can be obtained by substituting (4), (5) into (3):

$$
\begin{gathered}
(2 \mu+\lambda) \partial^{2} u_{1} / \partial x_{1}^{2}+\lambda \partial^{2} u_{2} / \partial x_{1} \partial x_{2}+(\mu+\alpha) \partial^{2} u_{1} / \partial x_{2}{ }^{2}+(\mu-\alpha) \partial^{2} u_{2} / \partial x_{1} \partial x_{2}+2 \alpha \partial \varphi_{3} / \partial x_{2}=0 \\
(\mu+\alpha) \partial^{2} u_{2} / \partial x_{1}^{2}+(\mu-\alpha) \partial^{2} u_{1} / \partial x_{1} \partial x_{2}+(2 \mu+\lambda) \partial^{2} u_{2} / \partial x_{2}{ }^{2}+\lambda \partial^{2} u_{1} / \partial x_{1} \partial x_{2}-2 \alpha \partial \varphi_{3} / \partial x_{1}=0 \\
B\left(\partial^{2} \varphi_{3} / \partial x_{1}{ }^{2}+\partial^{2} \varphi_{3} / \partial x_{2}{ }^{2}\right)+2 \alpha\left(\partial u_{2} / \partial x_{1}-\partial u_{1} / \partial x_{2}-2 \varphi_{3}\right)=0
\end{gathered}
$$

where $\lambda$ and $\mu$ are Lamé coefficients, $\alpha$ and $B$ are the Cosserat elastic moduli, the latter is bending stiffness. The Lamé coefficients $\lambda, \mu$ and the Cosserat parameters $\alpha$ and $B$ can be expressed through the micromechanical Cosserat model parameters, namely the solid volume fraction $v_{s}$, coordination number $k$, the sphere diameter $D$ and respective contact stiffnesses $k_{n}, k_{s}, k_{\varphi n}, k_{\varphi s}$ introduced in the previous section. This can be achieved by comparing micromechanical Cosserat elastic moduli (6), (7) in the constitutive equations (4) with corresponding terms in continuum Cosserat theory taken, for example from [6] that reads

$$
\begin{aligned}
& \sigma_{11}=(2 \mu+\lambda) \gamma_{11}+\lambda \gamma_{22}, \sigma_{12}=(\mu+\alpha) \gamma_{12}+(\mu-\alpha) \gamma_{21}, \sigma_{21}=(\mu+\alpha) \gamma_{21}+(\mu-\alpha) \gamma_{12}, \\
& \sigma_{22}=\lambda \gamma_{11}+(2 \mu+\lambda) \gamma_{22} \\
& \mu_{13}=B \kappa_{13}, \mu_{23}=B \kappa_{23}
\end{aligned}
$$

where the Cosserat deformation measures, strains $\gamma_{j i}$ and curvatures $\kappa_{j i}$, are given by (5).

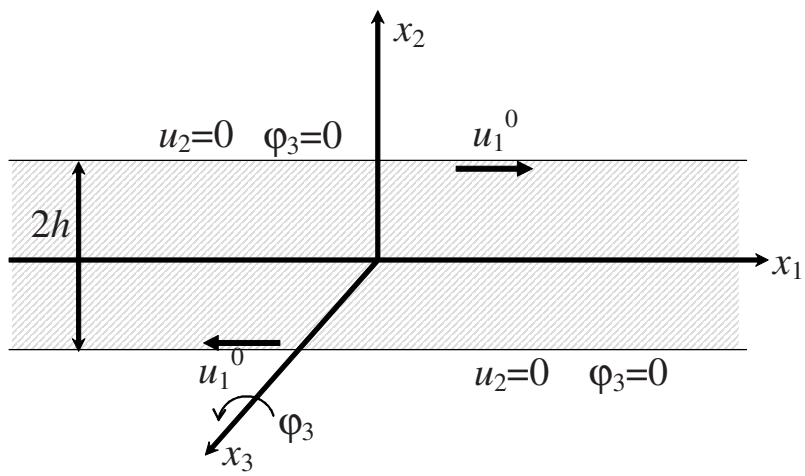

Fig. 1. A 2D model of fault with an elastic gouge with microrotations 
By doing so one obtains

$$
\begin{gathered}
\lambda=\nu_{s} k\left(k_{n}-k_{s}\right) /(5 \pi D), \mu=v_{s} k\left(2 k_{n}+3 k_{s}\right) /(10 \pi D), \alpha=v_{s} k k_{s} /(2 \pi D), \\
B=v_{s} k\left(k_{\varphi n}+4 k_{\varphi s}\right) /(5 \pi D) .
\end{gathered}
$$

Noting that the boundary conditions (8) are homogeneous along $x_{1}$, we are seeking a homogeneous along $x_{1}$ solution of the system of the Lamé equations (9-11) satisfying these boundary conditions. If we find such a solution according to the uniqueness theorem this solution will be unique.

Such a solution can be written in the normalized form $\left(h=1, u_{1}{ }^{0}=1\right)$ as follows:

$$
\begin{gathered}
u_{1}=\left(x_{2}-\frac{\sinh \left(x_{2} / l\right)}{\sinh (1 / l)} \Lambda^{2} l \tanh (1 / l)\right)\left(1-\Lambda^{2} l \tanh (1 / l)\right)^{-1}, u_{2}=0 \\
\varphi_{3}=-\frac{1}{2}\left(1-\frac{\cosh \left(x_{2} / l\right)}{\cosh (1 / l)}\right)\left(1-\Lambda^{2} l \tanh (1 / l)\right)^{-1}
\end{gathered}
$$

where

$$
\Lambda=l_{1} / l, \quad l=\left(l_{1}^{2}+l_{2}^{2}\right)^{1 / 2}, \quad l_{1}=(B / 4 \mu)^{1 / 2}, \quad l_{2}=(B / 4 \alpha)^{1 / 2} .
$$

Parameters $l_{1}$ and $l_{2}$ are two independent length scale parameters (normalized by $h$ ) reflecting the presence of a microstructure in the Cosserat continuum, with $l$ acting as a "hypotenuse" of the Cosserat continuum length scale parameters. Their expressions through the microstructural parameters by using (14) can be written as follows:

$$
\begin{gathered}
l_{1}=\frac{1}{2} \sqrt{\left(k_{\varphi_{n}}+4 k_{\varphi_{s}}\right) /\left(k_{n}+\frac{3}{2} k_{s}\right)} \quad l_{2}=\frac{\sqrt{2}}{2 \sqrt{5}} \sqrt{\left(k_{\varphi_{n}}+4 k_{\varphi_{s}}\right) / k_{s}} \\
l=\sqrt{\frac{k_{\varphi_{n}}+4 k_{\varphi_{s}}}{5 k_{s}} \cdot \frac{k_{n}+4 k_{s}}{2 k_{n}+3 k_{s}}} .
\end{gathered}
$$

Consequently, after further normalisation $(\mu=1)$ of stress and moment stress fields one obtains:

$$
\begin{gathered}
\sigma_{12}=\left(1-2 \Lambda^{2} \frac{\cosh \left(x_{2} / l\right)}{\cosh (1 / l)}\right)\left(1-\Lambda^{2} l \tanh (1 / l)\right)^{-1} \\
\sigma_{21}=\left(1-\Lambda^{2} l \tanh (1 / l)\right)^{-1},
\end{gathered}
$$




$$
\mu_{23}=2 \Lambda^{2} l \frac{\sinh \left(x_{2} / l\right)}{\cosh (1 / l)}\left(1-\Lambda^{2} l \tanh (1 / l)\right)^{-1}
$$

Figures 2-4 show the distributions of displacement and displacement gradient for various ratios of microstructural parameters $\Lambda, l$. The displacements (Fig. 2) lie between the one for the standard elastic solution $(\Lambda=0, l=0)$, and the Cosserat solution $(\Lambda=1, l=1)$ that exhibits maximum deviation from the standard continuum solution. The displacement gradient for relatively small values of the parameter $l$ (Fig. 3) exhibits a kind of plateau, which is insensitive to the values of the dimensionless parameter $0<\Lambda<1$. There is however a range of parameters $\Lambda$ and $l$, for which the displacement gradient is highly non-homogeneous and displays localisation reaching its peak for the combination of parameters $\Lambda=1, l=1$ (Fig. 4). Indeed, as the displacement gradient $u_{1,2}$ reaches its maximum at $x_{2}=0$ for any values of $\Lambda, l$, the parametric analysis of the solution performed for the middle layer of the gouge

$$
\left.u_{1,2}(\Lambda, l)\right|_{x_{2}=0}=\left(1-\frac{\Lambda^{2}}{\cosh (1 / l)}\right)\left(1-\Lambda^{2} l \tanh (1 / l)\right)^{-1}
$$

shows that displacement gradient takes its $\max$ at $\Lambda=1, l=1$. This combination of parameters corresponds to $l_{2}=0, l_{1}=l$. This means that the bending stiffness $B<<(\mu+\alpha) l^{2}$ the quantity in brackets being the effective shear modulus.

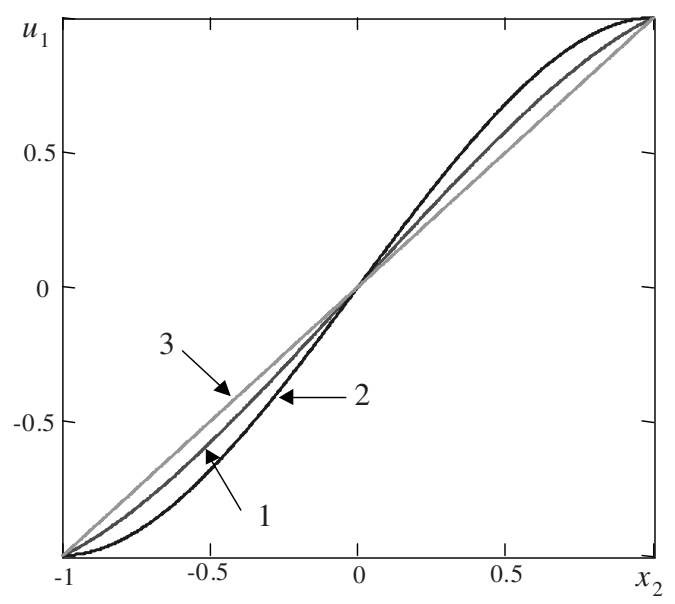

Fig. 2. Displacement distribution for various ratios of microstructural parameters $\Lambda, l$. Curve 1 corresponds to $\Lambda=0.8, l=0.5$, curve $2: \Lambda=1, l=1$, curve 3 : conventional elastic solution $\Lambda=0, l=0$ 


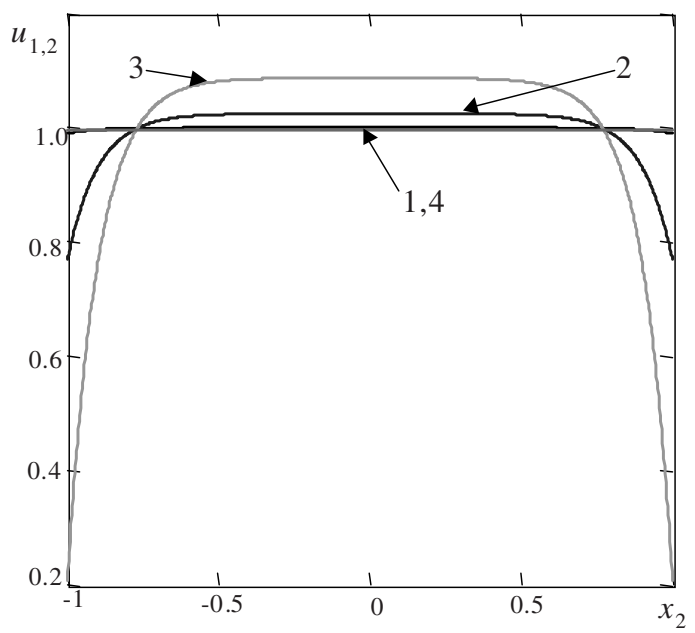

Fig. 3. Distribution of displacement gradients for small value of the parameter $l$ and various values of microstructural parameter $\Lambda$. Curve 1 corresponds to $\Lambda=0.1, l=0.1$, curve $2: \Lambda=0.5$, $l=0.1$, curve $3: \Lambda=0.9, l=0.1$, curve 4 : conventional elastic solution $\Lambda=0, l=0$

Figures 5 and 6 show the distributions of normalized moment stress $\mu_{23}$ and antisymmetric $\sigma_{[21]}$ and symmetric $\sigma_{(21)}$ parts of the shear stress. It is seen that the maximum values of the moment stress and the antisymmetric shear stress are attained at the gouge boundaries, while the maximum (localization) of the symmetric stress is achieved at the middle layer of the gouge the latter being in accordance with the localization of the displacement gradient.

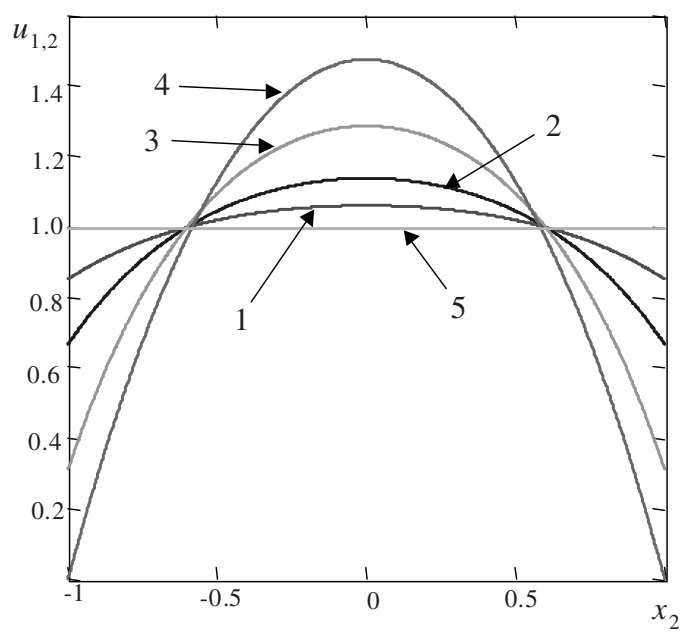

Fig. 4. Distribution of displacement gradients for large values of the parameter $l$ and various values of microstructural parameter $\Lambda$. Curve 1 corresponds to $\Lambda=0.5, l=0.5$, curve $2: \Lambda=0.7$, $l=0.5$, curve $3: \Lambda=0.9, l=0.5$, curve $4: \Lambda=1, l=1$, curve 5 : conventional elastic solution $\Lambda=0, l=0$ 


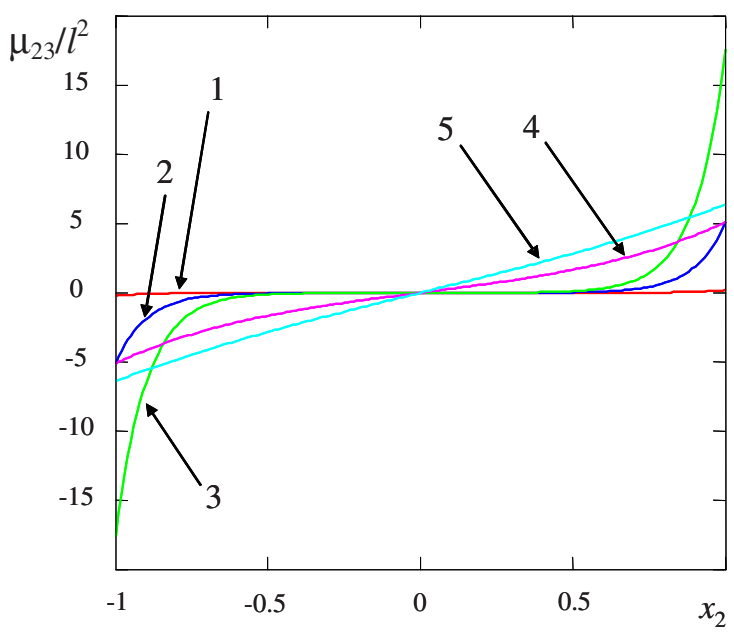

Fig. 5. Distribution of normalized moment stress for various values of the parameters $l$ and $\Lambda$. Curve 1 corresponds to $\Lambda=0.1, l=0.1$, curve $2: \Lambda=0.5, l=0.1$, curve $3: \Lambda=0.9, l=0.1$, curve 4 : $\Lambda=0.9, l=0.5$, curve $5: \Lambda=1, l=1$

(a)

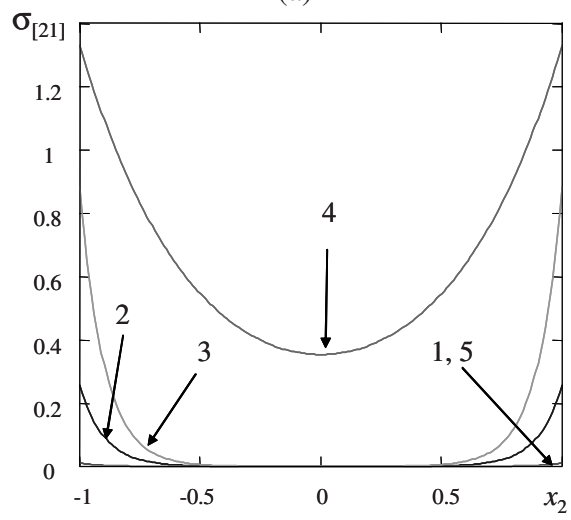

(b)

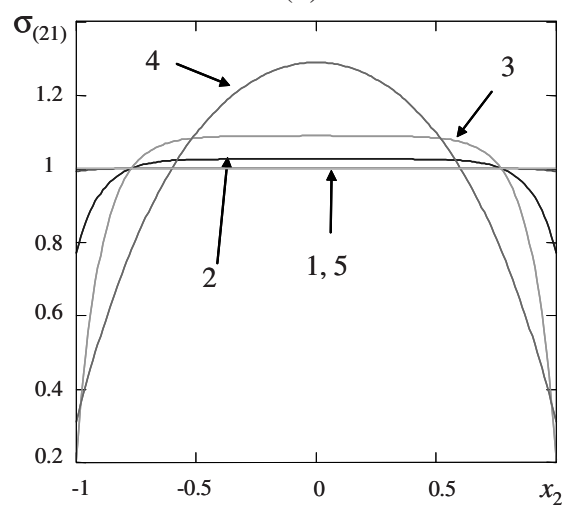

Fig. 6. Distribution of antisymmetric (a) and symmetric (b) parts of the shear stresses for various values of the parameters $l$ and $\Lambda$. Curves 1 correspond to $\Lambda=0.1, l=0.1$, curves $2: \Lambda=0.5$, $l=0.1$, curves $3: \Lambda=0.9, l=0.1$, curves $4: \Lambda=0.9, l=0.5$, curve 5 : conventional elastic solution $\Lambda=0, l=0$ 


\section{On the Possibility of Determining the Localization Regime from Wave Velocity Measurements}

Prior determination of whether the fault gouge can show localization requires the measurements of calculations of the Cosserat parameters, which is somewhat involved. It would be attractive to use the measurements of velocities of wave propagation through the gouge material for this purpose.

Consider propagation of planar waves in an infinite Cosserat material. According to [6] there are the following types of planar waves: (1) a conventional longitudinal wave, which is insensitive to the microrotations; (2) a twist wave which reflects the Cosserat properties of the material, but difficult to measure and; (3) one or two (depending upon frequency) shear waves. These shear waves show dispersion which is a result of the Cosserat properties and could therefore be used for identifying possible localization. The wave number $\xi$ for these shear waves satisfies the following characteristic equation (eg, [6])

$$
c_{2}^{2} c_{4}^{2} \xi^{4}+\left[\omega_{*}^{2} c_{5}^{2}-\omega^{2}\left(c_{2}^{2}+c_{4}^{2}\right)\right] \xi^{2}-\omega^{2}\left(\omega_{*}^{2}-\omega^{2}\right)=0
$$

where $\omega$ is the frequency and

$$
\omega_{*}^{2}=\frac{4 \alpha}{J}, \quad c_{2}^{2}=\frac{\mu+\alpha}{\rho}, \quad c_{4}^{2}=\frac{B}{J}, \quad c_{5}^{2}=\frac{\mu}{\rho}
$$

where $\rho$ is the material density, $J$ is the rotational inertia which in the case of material consisted of spherical grains of diameter $D$ is equal to $J=\rho D^{2} / 10$.

When $\omega<<\omega_{*}$ equation (22) gives only one solution which corresponds to the shear wave propagating with the velocity $\omega / \xi \cong c_{5}$ which is the conventional shear wave velocity. When $\omega=\omega_{*}$ equation (22) again gives only one solution corresponding to a shear wave traveling with velocity $c_{*}$, where

$$
c_{*}^{2}=\frac{(\mu+\alpha) B}{\alpha J+B \rho}
$$

Comparison of these two velocities with the aid of (23) and (17) leads to

$$
\frac{c_{*}^{2}}{c_{5}^{2}}=\frac{l^{2}}{l_{2}^{2}+0.025 D^{2}}=\frac{1}{1-\Lambda^{2}+0.025 D^{2} / l^{2}}
$$

If one assumes that the total characteristic length $l$ is at least not smaller than the particle diameter $D$, then the localization case, $\Lambda \rightarrow 1$ corresponds to the case of $c_{*} / c_{5}>>1$. Thus the localization case should correspond to considerable dispersion in the shear wave: its velocity should markedly increase with the increase in frequency. It should be noted that the waves at frequencies close to $\omega_{*}$ may considerably be attenuated by scattering since the length of the shear wave of frequency $\omega_{*}$ is 
$\lambda_{*}^{2}=0.025 D^{2} \Lambda^{-2} /\left(1+0.025 D^{2} / l_{2}^{2}\right)$ which, in the localization range $\Lambda \rightarrow 1$, is already below the particle diameter.

\section{Conclusion}

The presence of independent microrotations in particulate materials (e.g. the gouge) can lead to considerable localization of deformation gradients which when measured and interpreted in a sense of conventional medium create an impression of strain localization and associated material instability. Such a phenomenon, if experimentally confirmed will give a strong evidence of importance of the microrotational and Cosserat effects. The same phenomenon also leads to a considerable dispersion in shear wave propagation which can be used for independent experimental verification of the proposed mechanism of apparent strain localization in fault gouge.

Acknowledgment. The authors acknowledge the support of the UWA Small Research Grant 2001. The first author acknowledges the support of the ARC Discovery Grant DP0346148 and the Alexander von Humboldt Research Fellowship 2002-2003.

\section{References}

1. Rudnicki, J.W., Rice, J.R.: Conditions for the Localization of Deformation in PressureSensitive Dilatant Materials. J. Mech. Phys. Solids. 23 (1975) 371-394

2. Mühlhaus, H-B., Oka, F.: Dispersion and Wave Propagation in Discrete and Continuous Models for Granular Materials. Int. J. Solids Structures. 33 (1996) 2841-2858

3. Mühlhaus, H.-B., Dyskin, A.V., Pasternak, E., Adhikary D.: Non-Standard Continuum Theories in Geomechanics: Theory, Experiments and Analysis. In: Picu, R.C., Krempl, E. (eds.): Proc. Fourth International Conference on Constitutive Laws for Engineering Materials. Troy, New York (1999) 321-324

4. Pasternak, E., Mühlhaus, H.-B.: Cosserat and Non-Local Continuum Models for Problems of Wave Propagation in Fractured Materials, In: Zhao, X.L., Grzebieta, R.H. (eds.): Structural Failure and Plasticity (IMPLAST 2000). Pergamon, Amsterdam (2000) 741-746

5. Pasternak, E., Mühlhaus, H.-B.: Cosserat Continuum Modelling of Granulate Materials. In: Valliappan. S. and Khalili. N. (eds.): Computational Mechanics - New Frontiers for New Millennium. Elsevier Science, Amsterdam (2001) 1189-1194

6. Nowacki, W.: Theory of Micropolar Elasticity. Springer, Wien (1970) 